\title{
Optimisation De La Stérilisation Des Conserves De Poisson Par La Mise Ne Place De La Démarche Six Sigma DMAIC
}

\author{
Ismail Idrissi \\ Bouchra Benazzouz
}

Laboratoire de Génétique, Neuroendocrinologie et Biotechnologie, Faculté des Sciences, Université Ibn Tofail, Kénitra, Maroc

doi: 10.19044/esj.2017.v13n25p297 URL:http://dx.doi.org/10.19044/esj.2017.v13n25p297

\begin{abstract}
The highly competitive economic environment has prompted companies to improve their industrial performance. In this context, an agrifood company seeks to improve the performance of its production resources as well as the quality system through the implementation of continuous improvement projects. The object of this work is the presentation of the results of the process of the improvement of industrial performance by the implementation of the Six Sigma approach on a process of sterilization of canned fish. To this end, the deployment of this project has made it possible to reduce the sterilizing value by $30 \%$ in order to improve the organoleptic qualities of the product. Also, the total duration of the heat treatments has been reduced by $20 \%$, thus allowing a possible increase in productivity.
\end{abstract}

Keywords : Continuous Improvement, Six Sigma, Industrial Performance, Sterilization, Canned Fish

Résumé

L'environnement économique fortement concurrentiel a incité les entreprises agroalimentaires à améliorer leur performance industrielle du secteur de la pêche (Idrissi, 2017a). Dans ce contexte, une entreprise agroalimentaire, cherche à améliorer la performance de ses ressources de production ainsi que le système qualité à travers la mise en place de projets d'amélioration continue (Idrissi et al., 2016a).

L'objet de ce travail est la présentation des résultats du processus de l'amélioration de la performance industrielle par la mise en œuvre de la démarche Six Sigma sur un processus de stérilisation des conserves de poisson. A cet effet, le déploiement de ce projet a permet de réduire la valeur stérilisatrice de $30 \%$ afin d'améliorer les qualités organoleptiques du produit 
Egalement, la durée totale des traitements thermiques a été réduite de $20 \%$ permettant ainsi une possible augmentation de la productivité.

Mot clés : Amélioration continue, Six sigma, Performance industrielle, stérilisation, conserve de poisson

\section{Introduction}

Aujourd'hui déployé pour les gains en savoir-faire, savoir être et financiers, le Six Sigma s'est affirmé comme un facteur clé du développement des entreprises. La pérennité des solutions apportées en fait un gage de performance car les résultats obtenus sont concrets, démontrables et démontrés(Fréchet, 2005).

Les entreprises qui en ont compris la finalité et la force sont de plus en plus nombreuses à l'associer, de façon plus ou moins explicite, à leurs objectifs à moyen et long terme. Bien entendu, les méthodes et les applications diffèrent. Une partie des hésitations des entreprises pour mettre en place cette technique est d'ailleurs liée à la méconnaissance des impacts de la méthode dans leur contexte, et ceci qu'il s'agisse de services ou de production. L'une des clés de la méthode est l'application de la même démarche à tous les projets. Les résultats obtenus dépassent souvent les exigences posées en préambule et le niveau de qualité obtenu dépend de l'ensemble des outils utilisés. Dans cet article, la pratique des outils décrits dans la démarche Six Sigma tient une large place, sans rechercher l'exhaustivité sur les outils choisis ni sur leur utilisation. Le lien entre les outils et l'usage qui en est fait dans un projet spécifique est illustré au travers d'un exemple ponctué de conseils. J'ai réalisé, suivi et coaché des quantitésde projets. C'est pourquoi j'ai choisi de faire le lien entre la théorie du Six Sigma et les questions soulevées par sa mise en pratique, en proposant des réponses concrètes sur la réalisation d'un projet servant de fil conducteur. Lors de la réalisation d'un projet, on choisit parmi les outils à disposition ceux qui seront les plus pertinents en fonction de l'objectif visé, afin d'apporter des réponses concrètes. Les outils qui ont déjà fait leurs preuves et qui se trouvent agencés dans l'ordre imposé par la méthode Six Sigma ont un impact démultiplié et permettent alors de résoudre un grand nombre de problèmes. La démarche adoptée ici respecte le cycle DMAIC (Définir, Mesurer, Analyser, Innover, Contrôler). Le fil conducteur concret $\mathrm{du}$ projet permet de suivre les progrès accomplis. Les personnes qui cherchent des exemples avant de s'engager dans la mise en place de cette technique qui gagne maintes entreprises, des plus grandes (mondialement connues) aux PME-PMI, trouveront au travers de cette lecture une partie de leurs réponses. Dans l'article, la notion d'outils à utiliser pour la résolution d'un problème est présentée concrètement. Elle couvre à la fois des outils 
statistiques, de management et de communication. Certains paraissent simples, d'autres plus complexes. C'est pourquoi ils n'ont pas tous le même niveau d'utilisation, ni de présentation. J'ai simplement voulu montrer leur utilité dans un contexte de déploiement des projets.

L'originalité de l'article réside dans son plan, qui calque celui du déroulement d'un projet, sans négliger la pratique pouvant intéresser de nombreuses entreprises. Il aurait pu aussi être illustré par un autre sujet ou par un exemple dans les services ou couvrant un autre sujet. Tout d'abord, les principes de positionnement du projet concernant la description, le calcul de valeur ajoutée, les membres du groupe de travail, ainsi que le planning sont mis en place. La recherche des attentes du client s'effectue aussi dans la première partie. Dans la partie «Mesure», la démarche aboutit à l'identification des indicateurs contenus dans la problématique. Une grande partie des problèmes rencontrés dans les projets réside en effet dans la difficulté de mesurer : savoir quoi et comment mesurer sont des questions clés qui se posent au cours de cette phase. On examine d'abord ce que l'on va mesurer, puis on s'assure que l'on sait le faire. Dans la partie «Analyse», les causes des problèmes sont recherchées et la preuve qu'elles sont bien influentes déterminée. Différentes techniques sont alors appliquées. Dans la partie «Amélioration», les solutions déployées sont expliquées afin de remédier au problème. C'est à ce stade, et après les preuves de différences apportées dans la partie précédente, que les actions d'amélioration sont mises en place. Enfin, dans la partie «Contrôle», les différents éléments de la pérennisation, comme la formation et les outils de suivi (cartes de contrôle, standardisation, etc.) sont montrés. Ces éléments permettent de s'assurer que le nouveau processus, une fois mis en place, se maintiendra à son niveau actuel.La méthode Six Sigma pourrait paraître non innovante car elle utilise des outils qui ont déjà démontré leur puissance dans de nombreux cas. Cet article s'attache davantage à les articuler qu'à les expliciter pour s'intéresser à ce qui fait la force de la méthode, en particulier :

- l'écoute et l'intégration de la demande des clients;

- le soutien de la direction;

- l'utilisation d'outils statistiques dédiés à 1'amélioration du processus;

- la gestion de projet avec un cycle précis;

- les différents aspects de la communication;

- la prise en compte des aspects financiers des projets;

- l'appui sur une équipe de personnes formées et dédiées au déploiement de la méthode;

L'articulation de ces éléments permet d'apporter une réponse satisfaisante au plus grand nombre de problèmes. Cet article se veut accessible car l'idée n'est pas de devenir des experts de la méthode. Une réponse concrète à un projet d'amélioration et une marche à suivre logique 
pour y parvenir sont décrits ici, au travers d'un exemple suivi du début à la fin.

\section{PRÉSENTATION DE LA MÉTHODE SIX SIGMA.}

A. DÉFINITION DU SIX SIGMA : (pas nécessaire dans l'article)

Jusqu'à ce jour il n'existe aucune définition claire et complète de la méthode Six Sigma, cela est dû tout simplement à la question qui se pose souvent ' Qu'est ce que c'est Six Sigma ? Et qui nécessite plusieurs réponses vues sous plusieurs angles ("2006 ZINKGRAF - Six Sigma - The First 90 Days," n.d.). Cette définition se résume sur les 11 points suivants :

1. Six Sigma comme valeur : Le Six Sigma est avant tout une raison d'être pour l'entreprise, il se définit comme une stratégie ayant pour but d'améliorer en permanence les performances de l'entreprise. La stratégie ou valeur Six Sigma peut être illustrée dans la mission de l'entreprise et concerne aussi bien la recherche de l'excellence des produits, prestations et services proposés au client, mais aussi la recherche de l'excellence de la satisfaction des parties intéressées comme les actionnaires, les partenaires, les employés, ou les collectivités locales. Cette stratégie est principalement utilisée pour améliorer l'image de marque de l'entreprise, mais aussi pour se comparer (Benchmarking) et concurrencer les principaux concurrents leaders du marché. Mais, le Six Sigma est plus qu'une stratégie, il est aussi une culture qu'une entreprise doit faire adopter dans les différentes fonctions de son organisation, afin de sensibiliser tout le personnel des différentes niveaux hiérarchiques (George, 2004).

2. Six Sigma comme vision : Le Six Sigma peut s'articuler comme une vision à long terme, comme un objectif que l'entreprise cherche à obtenir dans le but d'atteindre l'excellence et d'accroitre les retours sur investissement de la mise en place du SMI entre autre, les investissements liés à la certification des normes qualité, d'hygiène, de sécurité, de santé et environnement, ainsi que des coûts engendrés par les actions d'améliorations et de contrôle.

3. Six Sigma comme approche managériale :L'application du Six Sigma dans une entreprise a pour but l'amélioration des critères, des exigences et des tolérances de la qualité concernant la satisfaction des clients, des employés, des actionnaires et de toute partie intéressée. Cette approche se déploie sous forme d'objectifs inscrits dans la stratégie et la politique QHSE de l'entreprise ; elle exige la mise en place de tout moyen et de toute action aidant à l'atteinte complète des objectifs signés par l'entreprise. L'approche managériale du Six Sigma aide l'entreprise à identifier et à éliminer les coûts cachés liés à la mise en place du système de management intégré, elle nécessite toute une réorganisation dans toutes les structures de l'entreprise avec une définition exacte des rôles et responsabilités de chaque acteur. La 
formation de nouvelles compétences est nécessaire à la réussite des projets Six Sigma lancés au niveau des différentes fonctions. Actuellement six sigma est appliquée par $88 \%$ des entreprises classées en top 25 par Forbes Magazine (Idrissi et al., 2016b) et par $7.27 \%$ des entreprises marocaines (Idrissi, 2017b)

4. Six Sigma comme mesure : La mise en place de projets Six Sigma dans une entreprise permet de mesurer et d'améliorer les défaillances et écarts par rapport aux objectifs de la politique QHSE. Le Six Sigma intègre les attentes implicites et explicites des clients et de toute partie intéressée comme exigence et spécification interne à respecter et à atteindre. Le Six Sigma est une mesure dans le sens qu'il permet de contrôler la performance des tableaux de bord, et d'être à son tour un indicateur significatif de mesure des variations et écarts cachés liés à un phénomène interne comme externe à 1'entreprise.

5. Six Sigma comme objectif : L'objectif du Six Sigma est l'atteinte d'un taux de défaut de 3,4 sur un million d'opportunités. Ceci représente 3,4 défauts sur un million de produits fabriqués, ou même 3,4 clients insatisfaits sur un million. Cet objectif vise l'excellence et la réduction de pertes financières liées aux défauts ou toute insatisfaction pour une qualité de $99,99966 \%$ sur les produits, services, satisfactions, performances, processus ou tous projets définis (George, 2002)

6. Six Sigma comme indicateur d'évaluation des performances : Le Six Sigma est considéré comme un indicateur car il permet d'évaluer les performances de l'entreprise. Pour cela, il évalue la capabilité des processus par rapport aux tolérances choisies par la direction; il évalue aussi la traçabilité des résultats souhaités en évaluant l'efficacité et l'efficience des ressources de l'entreprise par rapport aux objectifs de la politique QSE. Cette évaluation touche aussi le temps de cycle total d'exécution, de mise en œuvre et d'atteinte des objectifs, entre autres par l'évaluation du nombre de perte par million d'opportunité, ou par la perte de performance, qui est dans la plupart des cas causée par des facteurs cachés.

7. Six Sigma comme symbole : En statistique le symbole ' $\sigma$ ' représente l'unité de mesure de la variabilité dit 'écart type', cette mesure calcule la dispersion des valeurs autour de la moyenne ; le terme Six Sigma signifie donc "six fois l'écart type".

8. Six Sigma comme méthode : Le Six Sigma est une méthode de résolution des problèmes qui s'articule en 5 étapes (Définir, Mesurer, Analyser, Améliorer et Contrôler) nommée DMAIC. Cette méthode permet la réduction de la variation ou de tout écart par rapport à la cible souhaitée par l'entreprise, ou par rapport à la qualité $6 \sigma$ qui représente une efficacité de $99,99966 \%$ et une défaillance (écart- perte-défaut) de 0,00034\%. Cette méthode peut être appliquée sur n'importe quel problème tant qu'il y a des 
valeurs à mesurer et à analyser par rapport à leur cible. Elle englobe les défauts, la perte de temps, l'insatisfaction, le dysfonctionnement ou tout autre problème générant une perte de performance ou d'efficacité.

9. Six Sigma comme ensemble d'outils : La méthode Six Sigma emploie pour chaque étape de DMAIC des outils statistiques et managériaux afin de regrouper le maximum de données mesurables (tableaux de bord, des données chiffrées, des rapports) et de données subjectives (problèmes, analyses et idées d'amélioration proposées par une personne ou un groupe)

10.Six Sigma comme approche systémique : Le Six Sigma aborde dans son analyse une approche systémique en analysant la capabilité de chaque processus, la relation et l'interdépendance entre les différents processus, mais encore il analyse aussi la performance de l'ensemble du système de management intégré appliqué à l'entreprise.

11.Six Sigma comme qualité : Le Six Sigma représente aussi un symbole de la qualité , il entre dans les démarches d'améliorations continues du SMI. La qualité Six Sigma représente l'atteinte d'une qualité dite 'world class', ce qui veut dire qu'elle atteint une qualité irréprochable dépassant ainsi les exigences des normes ISO 9001, ISO 14001 et OHSAS 18001. Le concept qualité Six Sigma définit en luimême le pilier de l'excellence organisationnelle.

Toutes ces définitions peuvent être classées en trois grandes catégories ; la philosophie de Six Sigma, la mesure de Six Sigma et la méthode Six Sigma (Harry and Stewart, n.d.) La première catégorie définit le Six Sigmacomme une vision, une responsabilité et un engagement de l'entreprise visant à définir et satisfaire les exigences de toute partie intéressée. La deuxième catégorie le définit par contre comme un but, un niveau de performance, une mesure statistique et un benchmarking. L'unité de mesure utilisée visant à l'atteinte d'un taux de 3,4 défauts par million d'opportunités sert à comparer les processus, les unités de production, les concurrents directs, et même les compagnies proposant des produits de substitution. La troisième catégorie définit le Six Sigma comme une méthodologie, une approche systémique, un ensemble d'outils statistiques, une stratégie de percée et comme un moyen d'atteinte de la cible recherchée par le client ou de toute partie intéressée1. Ces trois catégories du Six Sigma peuvent être résumées dans une définition générale ; le Six Sigma est un processus d'activité qui permet aux entreprises d'améliorer radicalement leurs limites à travers une conception, une surveillance et un contrôle quotidien des processus de l'entreprise. Ces actions sont mises en valeur afin de minimiser les pertes en déchets ou en ressources en réduisant les erreurs liées aux ressources humaines, les défauts de qualité, ou tout écart existant entre la cible et le résultat du processus. Le Six Sigma fournit une méthode de recréation des processus qui encourage l'amélioration de la qualité et de la 
satisfaction des clients et de toute partie intéressée à travers une définition des objectifs de l'entreprise ainsi que leurs mesures de performance. Ces mesures sont utilisées pour améliorer les résultats des processus visant à accroitre les gains financiers. La méthode Six Sigma se présente sous forme de projet utilisant une exigence d'excellence pour une qualité, pour des outils statistiques, et pour un personnel compétent et qualifié.

La méthode Six Sigma est développée alors en 1986 par B. Smith aux ÉtatsUnis, en la définissant comme une démarche d'amélioration basée sur l'utilisation des outils statistiques (George, 2004) Le but de la mise en place de cette méthode était d'atteindre un objectif ambitieux jamais atteint auparavant qui est la minimisation des défauts à 3,4 DPMO (défauts par million d'opportunités)

\section{Description de la méthode DMAIC \\ 1 Étape 1 : Define}

\subsection{Définir le cadre du projet}

Après avoir entériné la décision de réaliser le projet, l'étape suivante à présent est d'en dessiner plus précisément les contours. La première étape d'une démarche DMAIC se décompose en trois parties :

- la préparation de la charte du projet. Il s'agit de compléter un document de synthèse décrivant le problème et les objectifs attendus, de constituer l'équipe et de sélectionner les parties prenantes, de reporter les enjeux financiers, enfin, de préparer la planification du projet;

- l'organisation du processus va faire l'objet d'un SIPOC (Suppliers, Input, Process, Output, Customers), en français FIPEC (Fournisseur, Intrants, Processus, Extrants, Clients). Le SIPOC reste le nom le plus connu;

- la synthèse de la voix du client pour en extraire les principaux éléments. Dans la phase de définition, il peut être intéressant de travailler sur un historique du problème.

\subsection{Préparer la charte du projet}

Le groupe de travail s'est constitué : au départ, le groupe est composé de personnes qui détiennent les principaux éléments du projet. Au fil du projet, la composition de l'équipe évolue afin d'intégrer des personnes plus opérationnelles pour restituer au propriétaire du processus, en fin de projet, un processus qui aura un fonctionnement efficace.

\subsection{L'organisation du processus : le SIPOC}

La réunion suivante permet de préparer la cartographie du processus, laquelle restera sommaire pendant la phase de définition. Les premiers éléments du SIPOC (Suppliers, Input, Process, Output, Customers) apparaissent. Le SIPOC trouve une improbable traduction dans FIPEC 
(Fournisseurs, Intrants, Processus, Extrants, Clients) le SIPOC du processus de stérilisation est présenté dans le chapitre « Résultats et discussion ».

\subsection{Synthétiser la voix du client}

L'étude de la voix du client nécessite plusieurs réunions, suivant le type de client ou bien le degré d'appréciation souhaité. En effet, lorsque le client dit : « Je ne veux pas acheter un produits insalubre (sous-stérilisé) ou caramélisé (sur-stérilisé) », il faut décoder cette attitude afin de comprendre ses attentes réelles.

Une part des informations cachées sous l'iceberg des coûts de nonqualité, dont on connaît rarement plus de $10 \%$ (Pillet, 2004), pourrait ainsi être révélée. Enfin, il faut comprendre ce que désire le client.

Atteindre 6 sigma symbolise le fait que, pour 1 million d'opportunités, seules 3,4 fois seront réalisées. Cela peut paraître peu, mais cela dépend des processus. Pour 10000000 de litres d'eau potable par an, un niveau de 6 sigma correspond à 34 litres d'eau non potable par an. Ce niveau de qualité est parfois largement atteint et dépassé. Mais, pour la majorité des entreprises, se situer à 2 ou 3 sigma sur les variables critiques est déjà une satisfaction (Fréchet, 2005).

Le nombre de sigma attendu évolue en fonction des exigences des clients (Fréchet, 2005). C'est pourquoi il est judicieux de bien identifier les différentes cibles auxquelles s'adressent les produits.

\section{2. Étape 2 : mesurer}

Cette étape est essentielle dans le déploiement de la démarche Six Sigma. Elle a pour objectif l'évaluation concrète de la performance des processus et leur adéquation aux demandes des clients. Autrement dit, son objectif est de renseigner, par les mesures appropriées sur le fonctionnement du processus par rapport aux exigences des clients

\section{3. Étape 3 : analyse du processus}

Les étapes 1 et 2 ont permis de dresser une cartographie du procédé afin d'identifier les causes (X potentiels) et de récolter des données à partir de moyens de mesure fiables. Il s'agit maintenant d'analyser ces données afin de déterminer les paramètres réellement influents dans la variabilité du processus. Tel est l'objectif de la phase d'analyse. Une fois ces paramètres déterminés, la phase d'innovation/ amélioration va permettre de développer la mise en œuvre de solutions pour stabiliser le processus à son meilleur niveau et de démontrer comment passer à la pratique, à un échelon souvent réduit. La phase de contrôle en fera une synthèse, en mettant en œuvre l'ensemble des améliorations apportées. 


\section{4. Étape 4 : innover/améliorer}

À la suite de la phase d'analyse, les principaux paramètres sont identifiés et l'on connait bien à présent les leviers qui permettront de ne plus avoir de conserves mal stérilisées. Mais s'il suffisait de connaître les paramètres importants pour atteindre les objectifs fixés, cela serait facile. En effet, même lorsqu'on connaît les causes de dysfonctionnement, un processus n'est pas maîtrisé pour autant.

Cette étape permet donc de passer de la théorie à l'application et de mettre en place des solutions aux améliorations détectées dans la phase d'analyse. C'est à ce stade également que les acteurs du processus vont s'impliquer davantage. Ils sont en effet les mieux placés pour connaître les problèmes en pratique, les interventions adaptées à réaliser sur le terrain et pour mettre en pratique au mieux les améliorations détectées lors des phases précédentes. L'étape d'innovation/amélioration consiste donc à trouver des solutions qui permettront de diminuer l'apparition des causes de problèmes identifiées à l'étape précédente

\section{5. Étape 5 : contrôler/maîtriser}

À la suite de la phase précédente, le travail sur les améliorations a déjà été testé et certaines ont été mises en place. Dans la phase de maîtrise du processus, toutes les améliorations trouvées au cours du projet sont intégrées au processus et l'on valide le fait que les nouveaux standards atteints satisfont bien les demandes du client. Dans le cadre de la maîtrise du processus, de nombreux éléments élaborés dans les phases précédentes sont synthétisés. Les modifications apportées au processus sont entérinées grâce à la validation des actions réalisées. Les aspects de documentation et de standardisation sont remis à jour avec les nouveaux éléments apportés. La variation des principaux paramètres, détectés durant la phase d'analyse, doit être maîtrisée et une carte de contrôle sur les éléments à maîtriser pourra, à l'issue du projet, être mise en place. La satisfaction du client fera l'objet de comparaisons pour déterminer les améliorations apportées au travers de la capabilité obtenue, en comparaison avec celle obtenue durant la phase de mesure. Cette étape de contrôle permet aussi de valider l'atteinte des objectifs financiers. Cette dernière phase du cycle DMAIC permet de restituer au Sponsor un processus qui présente un nombre de défauts très faible (quasi-inexistant). La réalisation du processus permet ainsi de maximiser les gains au niveau de la qualité des résultats obtenus et au niveau de l'atteinte des objectifs techniques et financiers.

\section{Capabilité Processus, interprétation}

Un processus est réputé capable si la dispersion de ses résultats est faible comparativement à la tolérance. 
Capabilité Processus : l'aptitude, la capacité d'un processus à respecter des spécifications, à atteindre en permanence le niveau de qualité souhaité. L'indice de Capabilité Processus $(\mathrm{Cp})$ fournit une indication sur la performance d'un processus par rapport aux limites admissibles, ce nombre sans unité indique le rapport entre la dispersion (la variabilité du processus) et la plage entre les tolérances. Plus grand ce nombre et plus le processus est "capable". Moins l'indice est élevé est plus la variabilité s'étend vers les limites, voire les dépasse.

On calcule la capabilité (notée $\mathrm{Cp}$ ) par le rapport : intervalle de tolérance / dispersion

$\mathrm{Cp}=$ avec $\mathrm{Ts}=$ tolérance supérieure, $\mathrm{Ti}=$ tolérance inférieure

Dans l'hypothèse du centrage de la dispersion entre les bornes des tolérances

\begin{tabular}{|c|c|c|c|}
\hline 1 & $\mathrm{Cp}>1.67$ & $\begin{array}{l}\text { Plus que } \\
\text { suffisant }\end{array}$ & $\begin{array}{l}\text { Non préoccupant, chercher à simplifier la gestion pour } \\
\text { réduire les coûts. }\end{array}$ \\
\hline 2 & $1.67>\mathrm{Cp}>1.33$ & Suffisant & Situation idéale. A maintenir. \\
\hline 3 & $1.33>\mathrm{Cp}>1.00$ & Trop juste & $\begin{array}{l}\text { Nécessite de l'attention, } \mathrm{Cp} \text { proche de } 1 \text { signifie qu'une } \\
\text { dérive peut créer des défauts. }\end{array}$ \\
\hline 4 & $1.00>\mathrm{Cp}>0.67$ & Insuffisant & $\begin{array}{l}\text { Existence de Non Conformes. il faut contrôler à } 100 \% \text {, } \\
\text { analyser le processus et si possible l'améliorer. }\end{array}$ \\
\hline 5 & $0.67>\mathrm{Cp}$ & $\begin{array}{l}\text { Très } \\
\text { insuffisant }\end{array}$ & $\begin{array}{l}\text { Analyse immédiate des causes, urgence de mise en } \\
\text { place de contre-mesures, révision des tolérances. }\end{array}$ \\
\hline
\end{tabular}

Tableau I Interprétation de la Capabilité Processus (Pekar, 1995)

\section{OPTIMISATION DU BAREME D'APPERTISATION DE L'AUTOCLAVE}

3.1 RAPPEL DU PROBLEME ET DE NOTRE OBJECTIF

Avant de déterminer le barème temps/température que nous avons retenu, rappelons en quelques mots le problème de la société étudiée ainsi que notre objectif. A quelques reprises des clients de La société étudiée ont signalé que les sardines se démêlaient trop lorsqu'ils faisaient réchauffer leur plat, c'est-à-dire que la viande se détachait des arêtes. Bien que cela n'altère en rien les qualités organoleptiques et sanitaires du produit, cela engendre quelques désagréments. En effet, les sardines dans une sauce, de ce fait en se détachant de l'arête en plusieurs petits morceaux, la viande s'imbibe plus facilement et plus rapidement de sauce. Par conséquent, elle devient moins présentable. 
4.2 Mise en place de la démarche DMAIC six sigma pour l'amélioration du procédé de stérilisation

I. Résultats, synthèse et discussion

A. Définir (Define)

\section{Charte de projet}

a. Informations Générales

\begin{tabular}{|l|l|}
\hline Société & Confidentielle \\
\hline Activités & Conserve de poisson \\
\hline Activité concernée & Stérilisation des conserves \\
\hline Processus concerné & Stérilisation \\
\hline Produits concernés & Sardines traditionnelles à l'huile \\
\hline Intitulé du projet & $\begin{array}{l}\text { Optimisation du processus de stérilisation des conserves par } \\
\text { application de la démarche Six Sigma DMAIC }\end{array}$ \\
\hline Manager du Projet & Ismail IDRISSI \\
\hline Adresse Email & $\underline{\text { ismail.idrissi@ @mail.com }}$ \\
\hline Numéro de téléphone & 0600611353 \\
\hline Date de Début du projet & $07 / 05 / 2016$ \\
\hline Date prévisionnelle de fin & $05 / 11 / 2016$ \\
\hline Coût estimé du projet & $71815,39 \mathrm{dhs}$ \\
\hline
\end{tabular}

\section{b. Description du problème, des buts, des objectifs et des livrables de ce projet}

\begin{tabular}{|c|c|}
\hline Problème & $\begin{array}{l}\text { - Les stérilisateurs sont sous exploités (taux de rendement } \\
\text { synthétique très bas) } \\
\text { - Le processus de stérilisation utilise un barème archaïque } \\
\text { et sur-stérilise les conserves des poissons. } \\
\text { - Les coûts de la non-qualité en stérilisation n'ont jamais } \\
\text { fait l'objet d'une étude pour les quantifier et les éliminer }\end{array}$ \\
\hline Objectif du projet & $\begin{array}{ll}\text { - } & \text { Optimisation du processus de stérilisation } \\
\text { - } & \text { Augmenter le chiffre d'affaire de la société } \\
\text { - } & \text { Eliminer les gaspillages en temps et main-d'œuvre } \\
\text { - } & \text { Augmentation du taux de rendement synthétique } \\
\end{array}$ \\
\hline
\end{tabular}

\section{c. Planification du projet}

Avant de se lancer dans la réalisation du projet, il est nécessaire de prendre le temps de découper celui-ci en tâches, afin de planifier leur exécution et le temps à allouer pour chacune d'elles.

Le tableau ci-dessous résume la planification de ce projet

\begin{tabular}{|l|l|l|l|l|l|}
\hline \multicolumn{2}{|l}{ Etape du projet } & Date début & Date fin & Durée \\
\hline Define & D & Définir & $07 / 05 / 2016$ & $19 / 05 / 2016$ & 12 Jours \\
\hline Mesure & M & Mesurer & $19 / 05 / 2016$ & $12 / 07 / 2016$ & 54 Jours \\
\hline Analyse & A & Analyser & $12 / 07 / 2016$ & $07 / 07 / 2016$ & 26 Jours \\
\hline Imporve & I & Améliorer & $07 / 07 / 2016$ & $06 / 09 / 2016$ & 61 Jours \\
\hline Control & C & Contrôler & $06 / 09 / 2016$ & $27 / 09 / 2016$ & 21 Jours \\
\hline
\end{tabular}




\section{d. Ressources attribuées au projet et budget prévisionnel}

Les ressources attribuées au projet et budget prévisionnel est résumé sur le tableau suivant :

\begin{tabular}{|l|l|l|l|l|}
\hline Département & Fonction & $\begin{array}{l}\text { Nombre d'heures de } \\
\text { travail } \\
\text { sur le projet }\end{array}$ & $\begin{array}{l}\text { Salaire } \\
\text { mensuel en } \\
\text { Dh }\end{array}$ & $\begin{array}{l}\text { Estimation du } \\
\text { coût en Dh }\end{array}$ \\
\hline Management Qualité & Directeur Qualité & 400 & 15000 & 28846 \\
\hline Management Qualité & $\begin{array}{l}\text { Responsable } \\
\text { assurance qualité }\end{array}$ & 221 & 8000 & 8500 \\
\hline Production & $\begin{array}{l}\text { Responsable } \\
\text { stérilisation }\end{array}$ & 300 & 5000 & 7211 \\
\hline Production & $\begin{array}{l}\text { Responsable } \\
\text { production }\end{array}$ & 32 & 10000 & 1538 \\
\hline $\begin{array}{l}\text { Location d'un dispositif ELLAB EVAL FLEX de validation et de suivi des barèmes } \\
\text { de stérilisation pour 2 jours }\end{array}$ & 10200,00 \\
\hline Coût total estimé en Dh & 56295 \\
\hline
\end{tabular}

\section{e. Diagramme SIPOC Pour le processus de stérilisation des conserves des sardines}

Le diagramme SIPOC (Suppliers-Inputs-Process-Outputs-Clients; Fournisseur-Entrées-Processus-sorties-Clients) Pour le processus de stérilisation des conserves des sardines est présenté au niveau du tableau suivant :

Tableau 1: Diagramme SIPOC Pour le processus de stérilisation des conserves des sardines

\begin{tabular}{|c|c|c|c|c|}
\hline Suppliers & Inputs & Process & Outputs & Clients \\
\hline Fournisseur & Entrées & Processus & sorties & Clients \\
\hline $\begin{array}{l}\text { Régiede } \\
\text { distribution de } \\
\text { l'eau potable }\end{array}$ & Eau propre & \multirow[t]{2}{*}{ Lavage } & \multirow[t]{2}{*}{$\begin{array}{l}\text { Eau et souillure } \\
\text { Boites lavées }\end{array}$} & \multirow[t]{2}{*}{$\begin{array}{l}\text { Département } \\
\text { de stérilisation }\end{array}$} \\
\hline RCI Industries & Détergent & & & \\
\hline $\begin{array}{l}\text { Département de } \\
\text { sertissage }\end{array}$ & Des Boites en vrac & $\begin{array}{l}\text { Mise } \\
\text { Paniers }\end{array}$ & $\begin{array}{l}\text { Paniers Pleines } \\
\text { de boites }\end{array}$ & $\begin{array}{l}\text { Département } \\
\text { de stérilisation }\end{array}$ \\
\hline $\begin{array}{l}\text { Département de } \\
\text { stérilisation }\end{array}$ & $\begin{array}{l}\text { Paniers } \\
\text { boites } \\
\text { stérilisées }\end{array}$ & Stérilisation & $\begin{array}{lr}\text { Paniers } & \text { Pleines } \\
\text { de } & \text { boites } \\
\text { stérilisées }\end{array}$ & $\begin{array}{l}\text { Département } \\
\text { de } \\
\text { refroidissement }\end{array}$ \\
\hline
\end{tabular}

\section{Les CTQ du processus de stérilisation}

Les Paramètres à optimiser sont appelés critiques pour la qualité (Critical to Quality) et sont présentés au niveau du tableau suivant : 
Tableau 3: Paramètre critique pour la qualité en processus de stérilisation

\begin{tabular}{|l|l|}
\hline Processus & $\begin{array}{l}\text { Paramètre critique pour la qualité } \\
\text { (à optimiser) }\end{array}$ \\
\hline \multirow{5}{*}{ Lavage } & $\begin{array}{l}\text { Température de lavage } \\
\text { Propreté boites } \\
\text { Coût main d'œuvre } \\
\text { Cadence de lavage }\end{array}$ \\
\hline \multirow{3}{*}{ Stérilisation } & Temps de destruction thermique TDT $\left(\mathrm{F}_{0}\right)$ \\
& Temps de stérilisation \\
& Taux de rendement synthétique \\
& Taux d'exploitation du stérilisateur \\
\hline
\end{tabular}

Tableau : Barème de stérilisation appliqué sur les deux stérilisateurs

\begin{tabular}{|c|c|c|c|}
\hline Etape & $\begin{array}{c}\text { Temps } \\
(\text { en } \text { min })\end{array}$ & $\begin{array}{c}\text { Température } \\
\left(\text { en }^{\circ} \text { C) }\right.\end{array}$ & $\begin{array}{c}\text { Pression } \\
\text { (en bar })\end{array}$ \\
\hline 1 & $\mathbf{8}$ & 90 & 1.2 \\
\hline 2 & 5 & 110 & 1.8 \\
\hline 3 & 3 & 123 & 1.8 \\
\hline 4 & 3 & 123 & 1.8 \\
\hline 5 & 1 & 122 & 1.8 \\
\hline 6 & 30 & 122 & 1.6 \\
\hline 7 & 5 & 100 & 1 \\
\hline 8 & 5 & 60 & 0 \\
\hline 9 & 15 & 40 & \\
\hline
\end{tabular}

\section{f. Description détaillée du produit étudié}

Description détaillée du produit étudié est présentée comme suite :

\begin{tabular}{|c|c|c|}
\hline \multirow{6}{*}{$\begin{array}{l}\text { Informations } \\
\text { générales }\end{array}$} & Nom & Sardines à l'huile de soja. \\
\hline & Espèce & Sardina pilchardus \\
\hline & Provenance du poisson & Poisson péché sur les côtes marocaines \\
\hline & Marque commerciale & $\begin{array}{l}\text { Marque client - Boite blanche sous étuis - } \\
\text { Boite lithographié }\end{array}$ \\
\hline & Format & 1/6P club OF \\
\hline & Mode de traitement & $\begin{array}{l}\text { Etêtage, équeutage, éviscération et } \\
\text { conditionnement manuel dans des boites } \\
\text { métalliques } \\
\text { Cuisson et stérilisation par traitement } \\
\text { thermique }\end{array}$ \\
\hline $\begin{array}{l}\text { Paramètres } \\
\text { physicochimiques }\end{array}$ & $\mathbf{p H}$ & 5,6 à 6,5 \\
\hline \multirow{2}{*}{$\begin{array}{l}\text { Paramètres } \\
\text { bactériologiques }\end{array}$} & $\begin{array}{l}\text { Résultat de l'étuvage à } 37^{\circ} \mathrm{C} \\
\text { pendant } 7 \text { jours }\end{array}$ & $\begin{array}{l}\text { Différence de ph inférieure à } 0.5 \text { par } \\
\text { rapport à la boite témoin }\end{array}$ \\
\hline & $\begin{array}{l}\text { Résultat de l'étuvage à } 55^{\circ} \mathrm{C} \\
\text { pendant } 7 \text { jours }\end{array}$ & $\begin{array}{l}\text { Différence de ph inférieure à } 0.5 \text { par } \\
\text { rapport à la boite témoin }\end{array}$ \\
\hline \multirow{4}{*}{$\begin{array}{l}\text { Paramètres } \\
\text { organoleptiques, }\end{array}$} & Odeur & $\begin{array}{l}\text { Sans rancissement, caractéristique de } \\
\text { l'espèce conditionnée }\end{array}$ \\
\hline & Texture & Caractéristique de l'espèce conditionnée \\
\hline & Gout & Caractéristique de l'espèce conditionnée \\
\hline & Aspect & Caractéristique de l'espèce conditionnée \\
\hline
\end{tabular}




\begin{tabular}{|c|c|c|c|}
\hline \multirow{5}{*}{ Composition } & Désignation & Pourcentage & Origine \\
\hline & Sel & 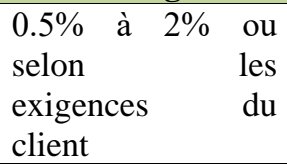 & Maroc \\
\hline & $\begin{array}{l}\text { Sardines équeutés étêtés } \\
\text { éviscérés. }\end{array}$ & $\begin{array}{lr}\text { Minimum } & 70 \% \text { ou } \\
\text { selon } & \text { les } \\
\text { exigences } & \text { du } \\
\text { client } & \\
\end{array}$ & Les cotes marocaines \\
\hline & $\begin{array}{l}\text { Huile de Soja entièrement } \\
\text { raffinée }\end{array}$ & $\begin{array}{l}28 \% \text { ou selon les } \\
\text { exigences du } \\
\text { client }\end{array}$ & $\begin{array}{l}\text { Origine du Maroc } \\
\text { et/ou UE }\end{array}$ \\
\hline & $\begin{array}{l}\text { Autres ingrédients (voir liste } \\
\text { des ingrédients) }\end{array}$ & $\begin{array}{l}\text { Avec ou sans } \\
\text { ingrédient } \\
\text { Quantité selon les } \\
\text { exigences du } \\
\text { client }\end{array}$ & $\begin{array}{l}\text { Origine du Maroc } \\
\text { et/ou UE }\end{array}$ \\
\hline \multirow{7}{*}{$\begin{array}{l}\text { Conditionnement, } \\
\text { stockage et } \\
\text { expédition }\end{array}$} & Emballage & \multicolumn{2}{|c|}{$\begin{array}{l}\text { Boites métallique illustrée en aluminium à } \\
\text { ouverture facile hermétiquement scellées } \\
\text { avec un vernis d'intérieure et d'extérieure } \\
\text { de qualité alimentaire } \\
\text { Cartons de } 50 \text { ou } 25 \text { boites, barquettes... et } \\
\text { selon les exigences du client }\end{array}$} \\
\hline & Etiquetage & \multicolumn{2}{|c|}{$\begin{array}{l}\text { Selon la réglementation marocaine et celle } \\
\text { de la destination du produit }\end{array}$} \\
\hline & Système d'emballage & \multicolumn{2}{|c|}{$\begin{array}{l}\text { Conditionnement en étui ou en barquette } \\
\text { sous atmosphère }\end{array}$} \\
\hline & Durée de Conservation & \multicolumn{2}{|c|}{$\begin{array}{l}6 \text { ans à partir de la date de fabrication (ou } \\
\text { moins selon les exigences des clients) à } \\
\text { température ambiante. }\end{array}$} \\
\hline & $\begin{array}{l}\text { Conditions de stockage sur } \\
\text { site }\end{array}$ & \multicolumn{2}{|c|}{$\begin{array}{l}\text { Manutention sans dommage } \\
\text { Stockage dans un milieu sec sans aucun } \\
\text { excès d'humidité à Température ambiante }\end{array}$} \\
\hline & $\begin{array}{l}\text { Conditions } \\
\text { transport/livraison }\end{array}$ & \multicolumn{2}{|c|}{$\begin{array}{l}\text { A manipuler avec soin pour éviter les } \\
\text { chocs entre les boites. }\end{array}$} \\
\hline & Marché de destination & \multicolumn{2}{|c|}{$\begin{array}{l}\text { Marché local } \\
\text { Marchés internationaux }\end{array}$} \\
\hline \multirow{4}{*}{$\begin{array}{l}\text { Utilisation } \\
\text { attendue } \\
\text { produit }\end{array}$} & Conseils d'utilisation & \multicolumn{2}{|c|}{$\begin{array}{l}\text { Produits consommés à froid ou à chaud } \\
\text { Mélangé avec d'autres aliments : salades } \\
\text { Consommation par toutes les catégories de } \\
\text { personnes à l'exception des nourrissons et } \\
\text { ce qui ont une allergie pour le poisson } \\
\text { Consommation directe après ouverture de } \\
\text { préférence }\end{array}$} \\
\hline & Personnes vulnérables & \multicolumn{2}{|c|}{$\begin{array}{l}\text { Les nourrissons et les personnes qui ont une } \\
\text { allergie pour le poisson }\end{array}$} \\
\hline & $\begin{array}{|lll|}\begin{array}{l}\text { Information } \\
\text { allergènes }\end{array} & \text { sur } & \text { les } \\
\end{array}$ & \multicolumn{2}{|c|}{ Ce produit contient du poisson } \\
\hline & Information sur les OGM & \multicolumn{2}{|c|}{ Ce produit ne contient pas d'OGM } \\
\hline
\end{tabular}




\section{B. Mesurer Mesure}

\section{Value Stream Mapping}

La Cartographie des flux de valeur Value Stream Mappingdes différentes étapes du processus de stérilisation est présentée comme suite :

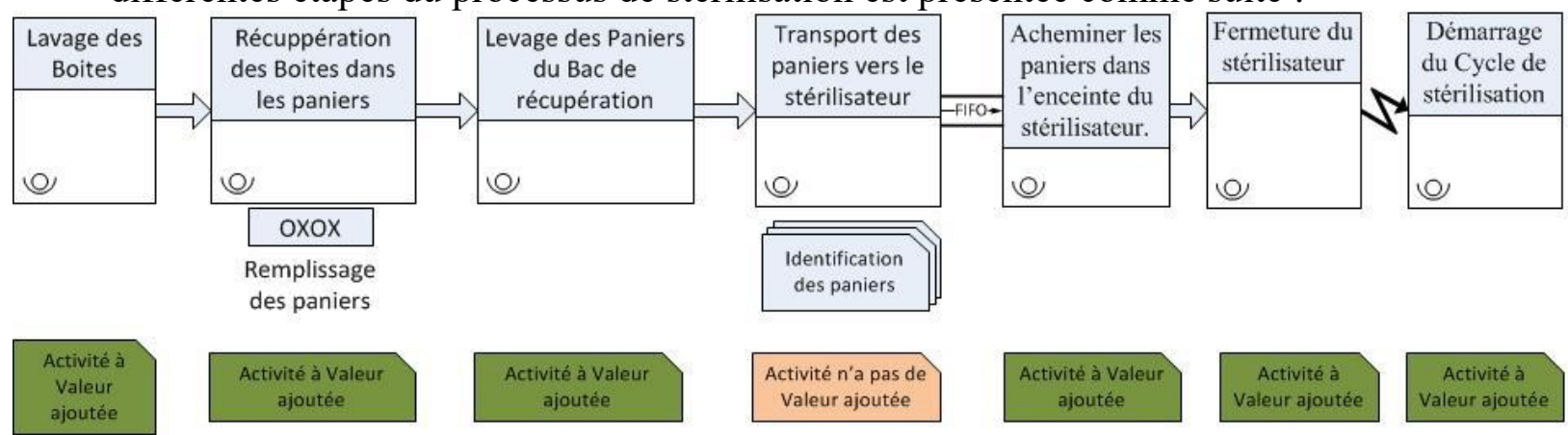

La Cartographie et l'analyse des flux de valeur a donné les résultats suivants :

$\underline{\text { Tableaux } 3 \text { : résultats de l'analyse des flux de valeur }}$

\begin{tabular}{|l|l|l|}
\cline { 2 - 3 } \multicolumn{1}{c|}{} & Nombre & Pourcentage \\
\hline Activités à valeur ajoutée & $\mathbf{7}$ & $\mathbf{8 7 . 5 \%}$ \\
\hline Activités qui n'ont pas de valeur ajoutée & $\mathbf{1}$ & $\mathbf{1 2 . 5 \%}$ \\
\hline
\end{tabular}

Une seule étape a été déterminée comme étant sans valeur ajoutée et doit être supprimée pour optimiser cette opération, la suppression de cette étape fera l'objet de la phase d'improve(améliorations).

\section{Suivi du taux du rendement synthétique et Taux d'exploitation des stérilisateurs}

\section{a. Stérilisateur 1}

Les résultats de Suivi du taux du rendement synthétique et Taux d'exploitation du stérilisateur $\mathrm{N}^{\circ} 1$ sont regroupés dans le tableau suivant : Tableau 4 : résultats de Suivi du taux du rendement synthétique et Taux d'exploitation du stérilisateur $\mathrm{N}^{\circ} 1$

\begin{tabular}{|l|l|l|l|l|l|l|l|}
\hline Stérilisateur & $\begin{array}{l}\text { Nombre } \\
\text { de cycles }\end{array}$ & $\begin{array}{l}\text { Nombre } \\
\text { théorique } \\
\text { des paniers }\end{array}$ & $\begin{array}{l}\text { Nombre } \\
\text { réel de } \\
\text { paniers }\end{array}$ & $\begin{array}{l}\text { Durée } \\
\text { d'utilisation } \\
\text { du } \\
\text { stérilisateur }\end{array}$ & $\begin{array}{l}\text { Durée } \\
\text { d'exploitation } \\
\text { du } \\
\text { stérilisateur }\end{array}$ & $\begin{array}{l}\text { Taux } \\
\text { d'exploitation } \\
\text { du } \\
\text { stérilisateur }\end{array}$ & $\begin{array}{l}\text { Taux de } \\
\text { rendement } \\
\text { synthétique }\end{array}$ \\
\hline $\mathbf{1}$ & $\mathbf{3}$ & $\mathbf{1 5}$ & $\mathbf{1 0}$ & $\mathbf{0 4 : 0 7}$ & $\mathbf{0 9 : 1 3}$ & $\mathbf{4 5 \%}$ & $\mathbf{6 7 \%}$ \\
\hline $\mathbf{1}$ & $\mathbf{5}$ & $\mathbf{2 5}$ & $\mathbf{1 8}$ & $\mathbf{0 6 : 5 0}$ & $\mathbf{1 3 : 1 5}$ & $\mathbf{5 2 \%}$ & $\mathbf{7 2 \%}$ \\
\hline $\mathbf{1}$ & $\mathbf{4}$ & $\mathbf{2 0}$ & $\mathbf{1 2}$ & $\mathbf{0 5 : 5 9}$ & $\mathbf{1 0 : 0 0}$ & $\mathbf{6 0 \%}$ & $\mathbf{6 0 \%}$ \\
\hline Max & $\mathbf{3}$ & $\mathbf{1 5}$ & $\mathbf{1 2}$ & $\mathbf{0 3 : 4 7}$ & $\mathbf{0 7 : 4 0}$ & $\mathbf{4 9 \%}$ & $\mathbf{8 0 \%}$ \\
\hline Min & $\mathbf{3}$ & $\mathbf{2 5}$ & $\mathbf{1 8}$ & $\mathbf{0 6 : 5 0}$ & $\mathbf{1 3 : 1 5}$ & $\mathbf{5 9 , 8 3 \%}$ & $\mathbf{8 0 , 0 0 \%}$ \\
\hline Moyenne & $\mathbf{3 , 7 5}$ & $\mathbf{1 5}$ & $\mathbf{1 0}$ & $\mathbf{0 3 : 4 7}$ & $\mathbf{0 7 : 4 0}$ & $\mathbf{4 4 , 6 7 \%}$ & $\mathbf{6 0 , 0 0 \%}$ \\
\hline
\end{tabular}


Le Taux moyen d'exploitation du stérilisateur 1 est de $51.35 \%$ ce qui est très bas et signifie que le stérilisateur est sous-exploité, ainsi Le TRS Taux moyen de rendement synthétique du stérilisateur 1 est de $69,67 \%$ ce qui signifie qu'il reste $30.33 \%$ du temps du stérilisateur à exploiter.

\section{a. Stérilisateur 2}

Les résultats de Suivi du taux du rendement synthétique et Taux d'exploitation du stérilisateur $\mathrm{N}^{\circ} 1$ sont regroupés dans le tableau suivant : Tableau 4 : résultats de Suivi du taux du rendement synthétique et Taux d'exploitation du stérilisateur $\mathrm{N}^{\circ} 2$

\begin{tabular}{|l|l|l|l|l|l|l|l|}
\hline $\begin{array}{l}\text { Stérilisate } \\
\text { ur }\end{array}$ & $\begin{array}{l}\text { Nomb } \\
\text { re de } \\
\text { cycles }\end{array}$ & $\begin{array}{l}\text { Nombr } \\
\text { e } \\
\text { théoriq } \\
\text { ue des } \\
\text { paniers }\end{array}$ & $\begin{array}{l}\text { Nomb réel } \\
\text { re } \\
\text { de } \\
\text { panier } \\
\text { S }\end{array}$ & $\begin{array}{l}\text { Durée } \\
\text { d'utilisati } \\
\text { on du } \\
\text { stérilisate } \\
\text { ur }\end{array}$ & $\begin{array}{l}\text { Durée } \\
\text { d'exploitati } \\
\text { on du } \\
\text { stérilisateu } \\
\text { r }\end{array}$ & $\begin{array}{l}\text { Taux } \\
\text { d'exploitati } \\
\text { on du } \\
\text { stérilisateu } \\
\text { r }\end{array}$ & $\begin{array}{l}\text { Taux de } \\
\text { rendeme } \\
\text { nt } \\
\text { synthétiq } \\
\text { ue }\end{array}$ \\
\hline 2 & $\mathbf{2}$ & $\mathbf{1 0}$ & $\mathbf{8}$ & $\mathbf{0 2 : 5 0}$ & $\mathbf{0 3 : 4 4}$ & $\mathbf{7 6 \%}$ & $\mathbf{8 0 \%}$ \\
\hline 2 & $\mathbf{4}$ & $\mathbf{2 0}$ & $\mathbf{1 3}$ & $\mathbf{0 5 : 5 7}$ & $\mathbf{1 2 : 0 1}$ & $\mathbf{5 0 \%}$ & $\mathbf{6 5 \%}$ \\
\hline $\mathbf{2}$ & $\mathbf{3}$ & $\mathbf{1 5}$ & $\mathbf{1 0}$ & $\mathbf{0 3 : 4 5}$ & $\mathbf{0 6 : 3 3}$ & $\mathbf{5 7 \%}$ & $\mathbf{6 7 \%}$ \\
\hline $\mathbf{2}$ & $\mathbf{2}$ & $\mathbf{1 0}$ & $\mathbf{8}$ & $\mathbf{0 2 : 5 7}$ & $\mathbf{0 5 : 1 3}$ & $\mathbf{5 7 \%}$ & $\mathbf{8 0 \%}$ \\
\hline Max & $\mathbf{4}$ & $\mathbf{2 0}$ & $\mathbf{1 3}$ & $\mathbf{0 5 : 5 7}$ & $\mathbf{1 2 : 0 1}$ & $\mathbf{7 5 , 8 9 \%}$ & $\mathbf{8 0 , 0 0 \%}$ \\
\hline Min & $\mathbf{2}$ & $\mathbf{1 0}$ & $\mathbf{8}$ & $\mathbf{0 2 : 5 0}$ & $\mathbf{0 3 : 4 4}$ & $\mathbf{4 9 , 5 1 \%}$ & $\mathbf{6 5 , 0 0 \%}$ \\
\hline Moyenne & $\mathbf{2 , 7 5}$ & $\mathbf{1 3 , 7 5}$ & $\mathbf{9 , 7 5}$ & $\mathbf{0 3 : 5 2}$ & $\mathbf{0 6 : 5 2}$ & $\mathbf{5 9 , 8 0 \%}$ & $\mathbf{7 2 , 9 2 \%}$ \\
\hline
\end{tabular}

Le Taux moyen d'exploitation du stérilisateur 1 est de $59,80 \%$ ce qui est très bas, ainsi le TRS Taux moyen de rendement synthétique du stérilisateur 1 est de $72,92 \%$ ce qui signifie qu'il reste $27.8 \%$ du temps du stérilisateur à exploiter.

\section{Suivi des Temps de Destruction Thermique sur les 12 sondes au niveau des stérilisateurs}

Les résultats de suivi des Temps de Destruction Thermique $\left(\mathrm{F}_{0}\right)$ sur les 12 sondes au niveau des stérilisateurs $1 \& 2$ sont présentés au niveau du tableau suivant :

Tableau 4 : Suivi des Temps de Destruction Thermique sur les 12 sondes au niveau des stérilisateurs

\begin{tabular}{|c|c|c|}
\cline { 2 - 3 } \multicolumn{1}{c|}{} & \multicolumn{2}{c|}{ Stérilisateur $\mathbf{N}^{\circ}$} \\
\cline { 2 - 3 } \multicolumn{1}{c|}{} & $\mathbf{1}$ & $\mathbf{2}$ \\
\hline $\mathbf{N}$ & $\mathbf{T D T}$ & $\mathbf{T D T}$ \\
\hline $\mathbf{1}$ & 7,20 & 19,56 \\
\hline $\mathbf{2}$ & 11,47 & 22,85 \\
\hline $\mathbf{3}$ & 7,30 & 21,46 \\
\hline $\mathbf{4}$ & 10,30 & 18,60 \\
\hline $\mathbf{5}$ & 17,87 & 18,43 \\
\hline $\mathbf{6}$ & 18,16 & 22,47 \\
\hline $\mathbf{7}$ & 15,41 & 26,91 \\
\hline $\mathbf{8}$ & 17,16 & 20,84 \\
\hline
\end{tabular}




\begin{tabular}{|c|c|c|}
\hline $\mathbf{9}$ & 35,40 & 23,58 \\
\hline $\mathbf{1 0}$ & 29,73 & 25,93 \\
\hline $\mathbf{1 1}$ & 31,10 & 19,75 \\
\hline $\mathbf{1 2}$ & 32,65 & 19,74 \\
\hline Min & 7,20 & 18,43 \\
\hline Max & 35,40 & 26,91 \\
\hline Ecart-type & 10,18 & 2,76 \\
\hline Moyenne & 19,48 & 21,68 \\
\hline
\end{tabular}

\section{Analyser Analyse}

Pour analyses les données collectées, on présente Grandeurs de la valeur stérilisatrice à appliquer en fonction des produits traités selon la littérature scientifique, voir tableau suivant :

Tableau : Grandeurs de la valeur stérilisatrice à appliquer en fonction des produits traités (source : (NICOLE and KNOCKAERT, 1989)

\begin{tabular}{|c|c|c|c|}
\hline Jus de couverture & Type de produit & $\mathrm{pH}>5,2$ & $4,5<\mathrm{pH}<5,2$ \\
\hline \multirow{2}{*}{$\begin{array}{l}\text { bactériostatique } \\
\text { (huile ou très salé) }\end{array}$} & Entier & $* \mathrm{TDT}>=3 \mathrm{~min}$ & $\begin{array}{l}* \mathrm{TDT}>=5 \\
\min \end{array}$ \\
\hline & $\begin{array}{l}\text { Broyé, en morceaux ou très } \\
\text { manipulé }\end{array}$ & $\begin{array}{l}* \text { TDT entre } 3 \text { min et } 5 \\
\text { min }\end{array}$ & $* \mathrm{TDT}>3 \mathrm{~min}$ \\
\hline \multirow[b]{2}{*}{ Eau ou sauce } & Entier & $* \mathrm{TDT}>=5 \mathrm{~min}$ & $* \mathrm{TDT}>3 \mathrm{~min}$ \\
\hline & $\begin{array}{l}\text { Broyé, en morceaux ou très } \\
\text { manipulé }\end{array}$ & $* \mathbf{T D T}>7 \mathrm{~min}$ & $* \mathrm{TDT}>5 \mathrm{~min}$ \\
\hline
\end{tabular}

**TDT : temps de destruction thermique $\mathbf{F}_{0}$

\section{Analyse des résultats de suivi sur le stérilisateur 1}

Les résultats d'Analyse des résultats de suivi des temps de destruction thermique sur le stérilisateur 1 sont présentés au niveau des deux tableaux suivants :

Tableau 2: Analyse des résultats de suivi des *TDT sur les 12 sondes au niveau du stérilisateur 1

\begin{tabular}{|c|c|c|}
\hline Paramètre & Valeur & Interprétation \\
\hline DDL (degré de liberté) & 12 & $\begin{array}{l}12 \text { sondes sont installées sur les boites au cours } \\
\text { de la stérilisation }\end{array}$ \\
\hline SCE (somme des carrés des écarts) & 1140,725 & \multirow{8}{*}{$\begin{array}{l}\text { Les *TDT différent significativement d'une } \\
\text { boite à une Autre }\end{array}$} \\
\hline Variance & 95,060 & \\
\hline Ecart-type & $\mathbf{1 0 , 1 8 3}$ & \\
\hline Min & 28,198 & \\
\hline Max & 35,400 & \\
\hline Ecart Minimal & 7,202 & \\
\hline Ecart Maximal & $-12,28$ & \\
\hline Etendu & 15,92 & \\
\hline Moyenne & 19,480 & \\
\hline
\end{tabular}

$\mathrm{Ci}$ aprèsl'analyse de capabilité du processus de stérilisation en stérilisateur 1 


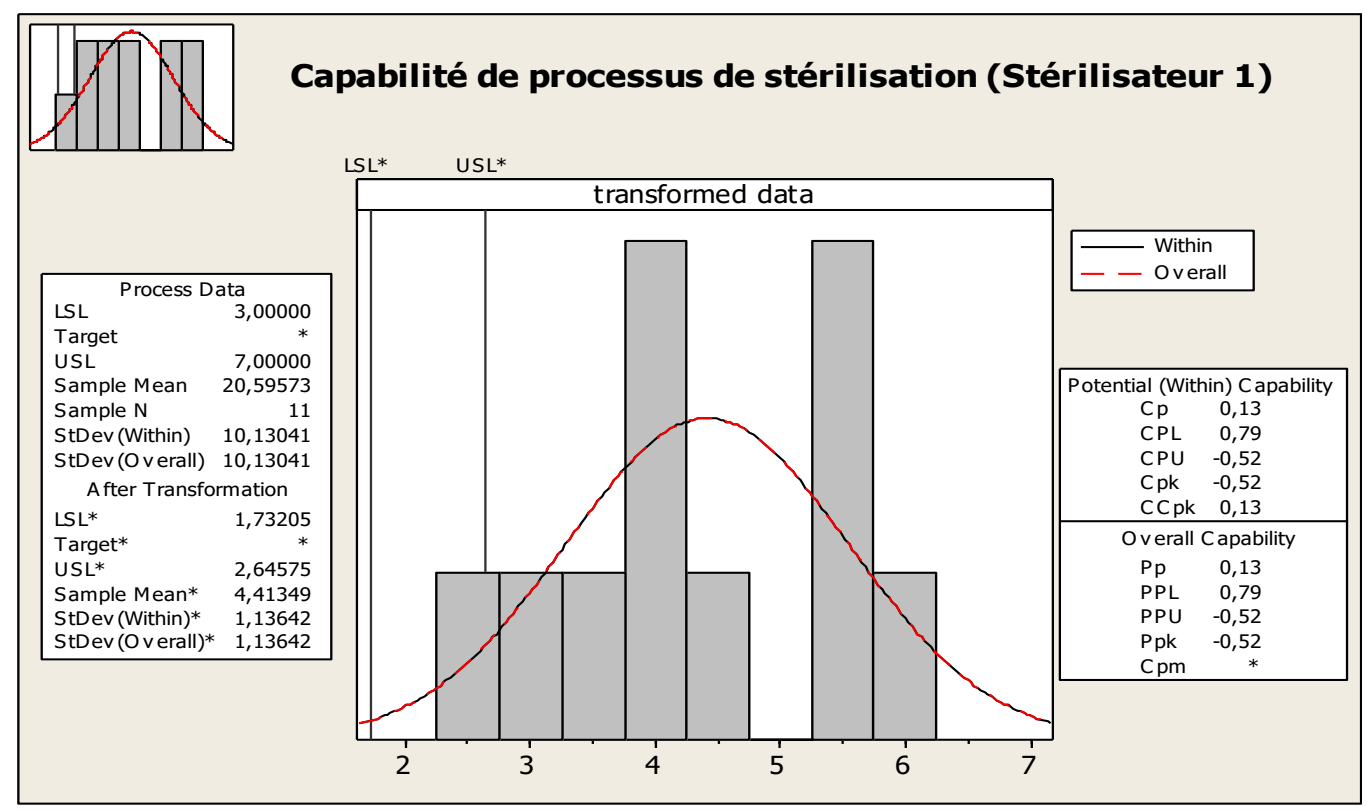

Selon le tableau des capabilités présentés ci avant (Pekar, 1995), il faut réaliser une analyse immédiate des causes. Le processus est Non Capable à satisfaire l'exigence du processus relative aux Grandeurs de la valeur stérilisatrice à appliquer pour le produit traité vu que la capabilité est inférieure à 0.67

\section{Analyse des résultats de suivi sur le stérilisateur 2}

Tableau 3 : Analyse des résultats de suivi des TDT sur les 12 sondes au niveau du stérilisateur 2

\begin{tabular}{|l|l|l|}
\hline Paramètre & Valeur & Interprétation \\
\hline $\begin{array}{l}\text { DDL (degré de de } \\
\text { liberté) }\end{array}$ & 12 & $\begin{array}{l}\text { 12 sondes sont installées sur les boites au cours de } \\
\text { la stérilisation }\end{array}$ \\
\hline $\begin{array}{l}\text { SCE (somme des } \\
\text { carrés des écarts) }\end{array}$ & $\mathbf{8 3 , 8 0 4}$ & \\
\hline Variance & $\mathbf{6 , 9 8 4}$ & \\
\cline { 1 - 2 } Ecart-type & $\mathbf{2 , 7 6 0}$ & \multirow{2}{*}{ Les *TDT différent significativement d'une boite à } \\
\cline { 1 - 2 } une Autre
\end{tabular}

Ci-après l'analyse de la capabilité du processus de stérilisation en stérilisateur 2 


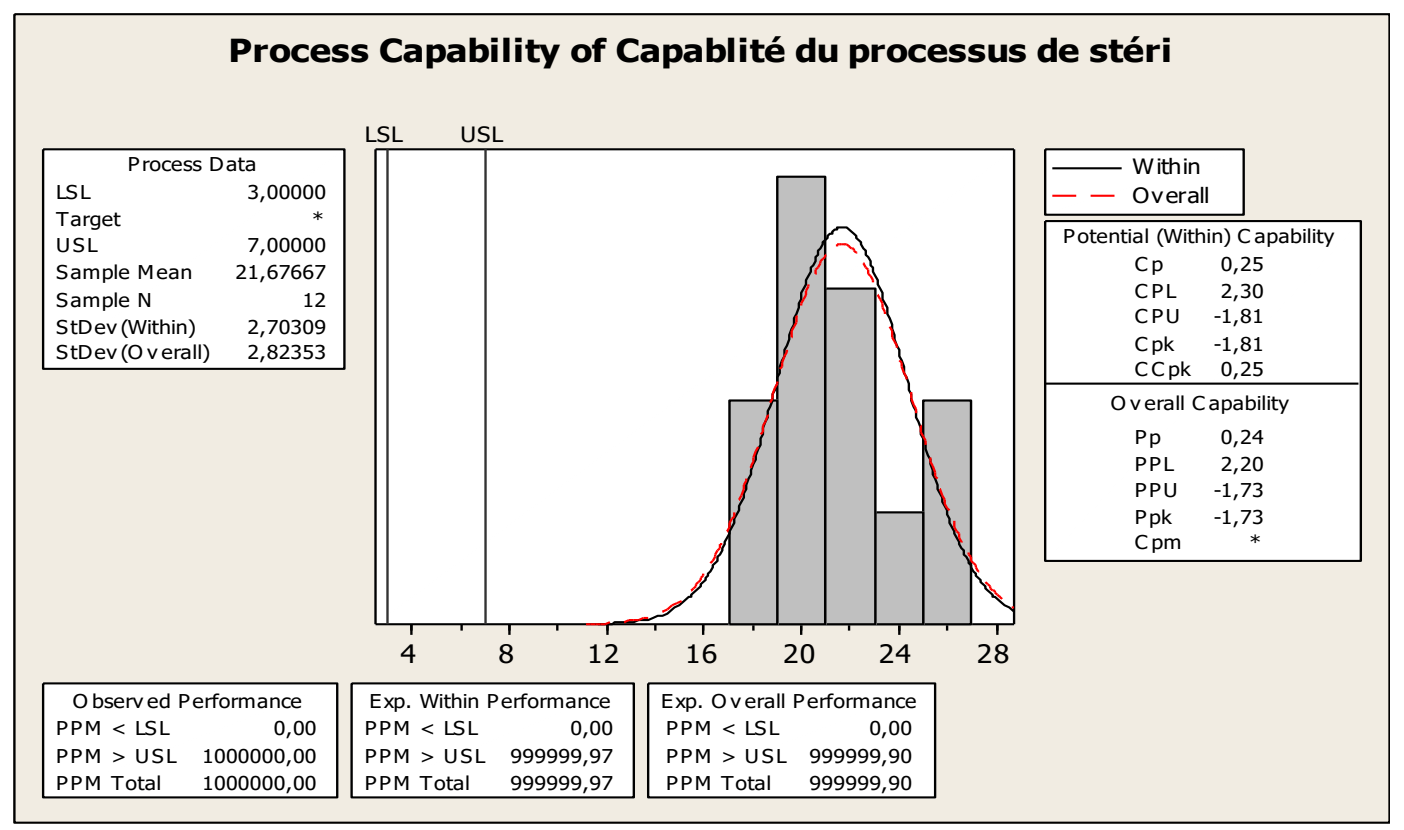

Selon le tableau des capabilités présentés ci avant (Pekar, 1995), il faut réaliser une analyse immédiate des causes. Le processus est Non Capable à satisfaire l'exigence du processus relative aux Grandeurs de la valeur stérilisatrice à appliquer pour le produit traité vu que la capabilité est inférieure à 0.67

\section{Mise en place des actions d'amélioration :}

Suite aux résultats d'analyse des processus de stérilisation sur les deux stérilisateurs de la société étudiée, les deux processus sont incapables de satisfaire aux exigences vu que les deux capabilités sont inférieures à 0.67 , pour cela nous avons améliorer les barèmes de stérilisation pour optimiser la capabilité, le barème suivant a été proposé :

Tableau : Barème de stérilisation proposé pour optimiser la capabilité du processus de stérilisation

\begin{tabular}{|c|c|c|c|}
\hline Etape & $\begin{array}{c}\text { Temps } \\
(\text { en } \text { min })\end{array}$ & $\begin{array}{c}\text { Température } \\
\left(\text { en }^{\circ} \text { C) }\right.\end{array}$ & $\begin{array}{c}\text { Pression } \\
(\text { en bar })\end{array}$ \\
\hline 1 & 8 & 90 & 1.2 \\
\hline 2 & 5 & 110 & 1.8 \\
\hline 3 & 3 & 123 & 1.8 \\
\hline 4 & 3 & 123 & 1.8 \\
\hline 5 & 1 & 122 & 1.8 \\
\hline 6 & 20 & 122 & 1.8 \\
\hline 7 & 5 & 100 & 1.6 \\
\hline 8 & 5 & 60 & 1 \\
\hline 9 & 10 & 40 & 0 \\
\hline
\end{tabular}


Ce nouveau barème proposé, est à 20 minutes de temps de palier au lieu de 30 minutes dans l'ancien barème et 10 minutes de temps de refroidissement au lien de $15 \mathrm{~min}$, ce qui nous fera gagner $15 \mathrm{~min}$ par cycle et par stérilisateur, avec les temps de destruction thermiques suivant :

\begin{tabular}{|c|c|}
\hline $\mathbf{N}^{\circ}$ de sonde & TDT \\
\hline $\mathbf{1}$ & 5,0 \\
\hline $\mathbf{2}$ & 8,0 \\
\hline $\mathbf{4}$ & 6,1 \\
\hline $\mathbf{5}$ & 7,2 \\
\hline $\mathbf{6}$ & 11,5 \\
\hline $\mathbf{7}$ & 12,7 \\
\hline $\mathbf{8}$ & 10,8 \\
\hline $\mathbf{9}$ & 12,0 \\
\hline $\mathbf{1 0}$ & 24,8 \\
\hline $\mathbf{1 1}$ & 20,8 \\
\hline $\mathbf{1 2}$ & 21,8 \\
\hline Min & 22,9 \\
\hline Max & 5,0 \\
\hline Ecart-type & 24,8 \\
\hline Moyenne & 7,1 \\
\hline
\end{tabular}

Ces valeurs de TDTs sont relatifs au stérilisateur 1, on remarque les valeurs sont restées très dispersées (Ecarttype=7.1) car les paniers des boites sont remplies en vrac.

La valeur stérilisatrice minimale est de 5 minutes ce qui respecte l'exigence de stérilisation, la valeur maximale est de 24.8 minutes avec une moyenne de 13.6 , cette grande dispersion peut être évitée par le rangement et l'organisation des boites par des intercalaires au niveau des paniers.

\section{Conclusion :}

Le barème de stérilisation proposé est applicable à l'échelle industrielle. Il permet de réduire la valeur stérilisatrice de $30 \%$ afin d'améliorerles qualités organoleptiques du produit Egalement, la durée totale des traitements thermiquesa été réduite de $20 \%$ permettant ainsi unepossible augmentation de la productivité.

\section{References:}

1. 2006 ZINKGRAF - Six Sigma - The First 90 Days, n.d.

2. Fréchet, C., 2005. le Six Sigma.

3. George, M., 2004. 2004 GEORGE - What is Lean Six Sigma.

4. George, M., 2002. Lean Six Sigma: Combining Six Sigma Quality with Lean Production Speed 300. doi:10.1036/0071385215

5. Harry, M.J., Stewart, R., n.d. Six Sigma Mechanical Design Tolerancing. Mororola Inc.

6. Idrissi, I., 2017a. Le secteur de la peche au Maroc Un secteur en pleine expansion.

7. Idrissi, I., 2017b. Etude du niveau d'implémentation de la Démarche 
Lean Six Sigma au niveau des entreprises marocaines 27-28.

8. Idrissi, I., Aftais, I., Mesfioui, A., Benazzouz, B., 2016a. Food Processing Optimization using Lean Six Sigma Methodology - Case Study of a Mackerel Filets Production Company in Morocco 143, 273-281.

9. Idrissi, I., Aftais, I., Mesfioui, A., Benazzouz, B., 2016b. Analysis of Relation Between Financial Performance and the Use of Lean Six Sigma by the Top Fortune Companies Worldwide as Published by Forbes Magazine 64-66.

10. NICOLE, J.P., KNOCKAERT, C., 1989. Les conserves des produits de la mer 159 p. : ill., graph.

11. Pekar, J., 1995. Total quality management: Guiding principles for application.

12. Pillet, M., 2004. Six Sigma. 